\title{
Dynamical Equivalent Circuit for 1-D Periodic Compound Gratings
}

\author{
Carlos Molero, Raúl Rodríguez-Berral, Francisco Mesa, Fellow, IEEE, and Francisco Medina, Fellow, IEEE
}

\begin{abstract}
Metallic compound gratings are studied in this work by means of an analytical equivalent circuit approach in order to obtain its transmission and reflection properties when illuminated by a TM-polarized plane wave. A compound grating consists of the periodic repetition of a finite number of slits carved out of a thick metal slab (reflection grating) or connecting two separated open regions through groups of slits in the metal slab (transmission grating). The equivalent circuit is rigorously obtained starting from a simplified version of the integral equation for the electric field at the slits apertures. That equivalent circuit involves transmission-line sections that account for the fundamental and lowest order diffracted modes (which does give the "dynamical" nature to the present equivalent circuit), and lumped components to model the effect of all the higher order diffracted modes. All the relevant and complex features of the spectra can be satisfactorily explained in terms of the topology and characteristics of the equivalent circuit. In contrast with some previously reported circuit models, all the dynamical and quasi-static circuit elements are analytically and explicitly obtained in terms of the geometric and electrical parameters of the grating. The accuracy of the approximate circuit model is very good over a very wide band, as it is demonstrated by comparison with full-wave data computed with commercial electromagnetic solvers.
\end{abstract}

Index Terms-Compound gratings, electromagnetic modeling, equivalent circuits, periodic structures, scattering problems.

\section{INTRODUCTION}

A LTHOUGH THE study of the optical and electromagnetic properties of periodically structured metallic surfaces has a long tradition [1]-[4], the interest on the subject was recently spurred by the discovery of the extraordinary optical transmission (EOT) at the end of the 1990s [5]. Since then, hundreds of papers have been devoted to the explanation of the physical grounds of such phenomenon and to the study of a variety of situations where some exotic behaviors can be observed. Several

Manuscript received September 27, 2015; revised January 19, 2016; accepted February 12, 2016. Date of publication March 01, 2016; date of current version April 01, 2016.This work was supported by the Spanish Ministerio de Economía y Competitividad and European Union FEDER funds under Project TEC201341913-P and by the Spanish Consejería de Innovación, Ciencia y Empresa, Junta de Andalucía under Project P12-TIC-1435.

C. Molero, R. Rodríguez-Berral, and F. Mesa are with the Microwaves Group, Department of Applied Physics 1, ETS de Ingeniería Informática, University of Sevilla, 41012 Seville, Spain (e-mail: cmolero@us.es; rrberral@us.es; mesa@us.es).

F. Medina is with the Faculty of Physics, Department of Electronics and Electromagnetism, University of Sevilla, 41012 Seville, Spain (e-mail: medina@us. es).

Color versions of one or more of the figures in this paper are available online at http://ieeexplore.ieee.org.

Digital Object Identifier 10.1109/TMTT.2016.2531663 authoritative papers have been published reporting comprehensive and exhaustive reviews about this topic [6]-[9]. Most of the material published during the last 15 years deals with 2-D arrays of holes in metal or dielectric screens. However, the study of the properties of 1-D periodic systems (such as arrays of narrow slits or strips in metal screens) has also been carried out in the frame of the analysis of EOT phenomena [10], [11]. Indeed, extraordinary transmission through free-standing infinite arrays of closely spaced metallic bars can be appreciated in the transmission curves published in a couple of Ukrainian papers [12], [13] almost 50 years ago. In spite of this long interest, the study of 1-D periodic gratings is an attractive research topic even today [14]-[26].

Roughly speaking, EOT and other mechanisms of enhanced transmission (such as Fabry-Pérot (FP) resonances occurring in 1-D periodic distributions of slits in thick metal gratings) have been linked to the interaction of impinging uniform planar waves with the so-called spoof (or designer) plasmons [27], [28], which basically are surface waves supported by periodically structured surfaces (for the 1-D periodic case the reader can be referred to the well-known Collin textbook on guided waves [29]). This point of view directly connects the transmission or reflection spectra with the dispersion diagrams of the surface/leaky modes. However, the obtaining of these dispersion diagrams is generally more cumbersome than directly computing the transmission or reflection curves. Fortunately, at least for frequencies well below the optical regime (when metals can be modeled as perfect or quasi-perfect conductors), an alternative model was proposed some years ago leading to a circuit-like description that explains the measured or calculated enhanced transmissivities [30]. The methodology originally introduced in [30] was later applied to a number of periodic structures of interest in the fields of optics (metal gratings) and microwave technology (frequency-selective surfaces, artificial magnetic conductors, and so on). Some instances of this research can be found in [14], [16], and [31]-[35], and an interesting overview focused on frequency-selective surfaces has recently been reported in [36]. Metallic compound gratings are interesting examples of systems that have attracted the attention of researchers in the past [12], [13], [37] and more recently in [38]-[45]. The interest on this kind of structures mainly lies on their rich-full electrical responses. The cross sections of these 1-D periodic structures are sketched in Fig. 1(a) and 1(b) for the reflection- and transmission-type versions, respectively. They exhibit extremely narrowband phase resonances due to the interactions between neighbor slits, which are accompanied by a 


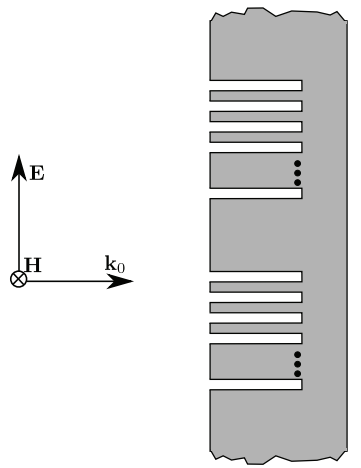

a)

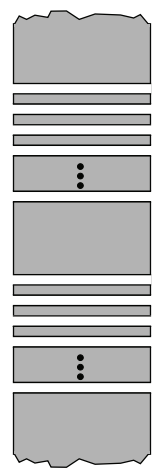

b)
Fig. 1. Cross sections of the type of structures analyzed in this paper. (a) Reflection compound grating and (b) transmission compound grating. The groups of slits are periodically repeated along the vertical direction. The slits might be different in width, depth, and filling, but each unit-cell group must have an horizontal symmetry plane.

strong magnetic field enhancement at the apertures [46]-[48] that depend on the groove depth and size and the distance between cavities.

During the last few years, accurate circuit models for the simplest case involving a single slit per period have been developed in [14] and [49]-[51]. Those models incorporate dynamical effects in such a way that the extraordinary transmission and the diffraction regimes are taken into account very accurately. However, to the authors' knowledge, no dynamical circuit model has been proposed for the more interesting case of compound grating involving three or more slits per period [41]. A quasi-static analytical circuit model was proposed by some of the authors in [14]. However, the derivation of that circuit made use of a rather heuristic approach that proposed a network of edge capacitors to approximately incorporate the interactions (through diffracted fields) between the transmission lines that modeled the slits in the unit cell. These interactions were shown to be essential to understand the behavior of the structure, especially to explain the appearance of deep narrowband transmission dips inside the FP transmission bands. Those dips had been explained before in terms of the so-called phase resonances [41]. Due to the quasi-static nature of the equivalent circuit in [14], our results were expected to be accurate provided the transmission dips took place at frequencies well below the onset of the diffraction regime (marked by the first Rayleigh-Wood (RW)'s anomaly frequency point). Actually, the model qualitatively predicted the existence of the transmission dips, but its quantitative results worsen considerably at frequencies relatively close to the onset of such a regime. Obviously, the quasi-static model could not take into account the operation of the structure beyond the first RW anomaly. Interestingly, reflection-like compound gratings exhibit their most relevant effects (specular reflection points associated with the phase resonances) within the diffraction regime [38], [39], i.e., at frequencies above the onset of the first RW anomaly. The analytical model proposed in [14] was then meaningless to explain the most interesting properties of the compound reflection gratings. The purpose of this paper is to improve the circuit model presented in [14] so as to take into account the dy-

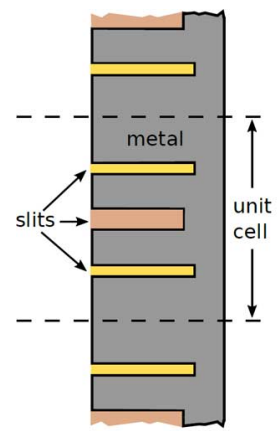

(a)

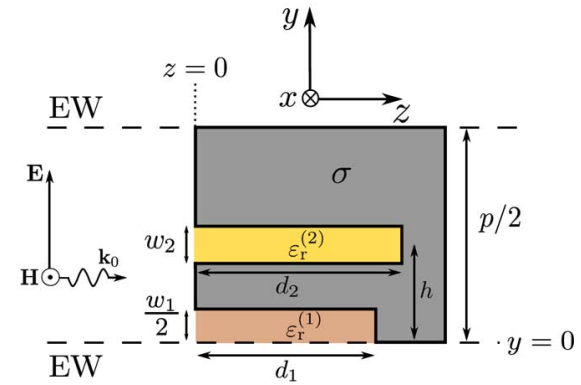

(b)
Fig. 2. (a) Example of a three-grooves per period reflection structure. This is the simplest symmetrical compound grating exhibiting phase resonances under normal TM incidence conditions. (b) Half unit cell bounded by virtual electric walls. The period of the structure is $p ; d_{i}$ is the groove depth of the $i$ th cavity $(i=1,2) ; h$ is the distance from the horizontal symmetry plane to the center of the side slits; $w_{i}$ is the slit width; $\varepsilon_{\mathrm{r}}^{(i)}$ are the relative permittivities of the media filling the slits; $\sigma$ is the conductivity of the metal.

namical high-frequency effects. The details of the derivation of the dynamic circuit model using an ab initio approach are presented in Section II. Apart from making it possible to explore the electrical response within the diffraction regime, the enhanced model will yield more accurate results below the diffraction regime, including extraordinary transmission effects for the compound grating case that were not accounted for by the quasistatic model in [14]. In particular, the interesting results reported in [38] and [39] can now be explained by the analytical model. Several numerical studies confirming the suitability of the proposed model are provided in Section III. Note that all the electrical parameters appearing in the proposed circuit model are analytically derived, in contrast with the calculation of the edge capacitances in [14], which were numerically computed using a finite-element Laplace's solver. The accuracy of the results obtained with our circuit model have been systematically checked against numerical data generated with a commercial full-wave solver or against experimental results, when available.

\section{Derivation of the Circuit Model}

Let us consider a 1-D compound grating with a symmetrical unit cell composed by three slits per period. An example of a reflection grating with these characteristics is sketched in Fig. 2(a). This is the simplest symmetrical 1-D compound grating exhibiting phase resonances under normal incidence [14], [41]. The structure is illuminated with a plane wave at normal incidence and with its electric field polarized along the $y$-direction (perpendicular to the slits). As a consequence of the invariance along the $x$-direction and the polarization of the incident wave, only TM fields are present. (The TE problem could have been considered by following very similar lines. Actually this TE problem was treated in [49] for simple gratings, but it has no major interest in the context of the present study of compound gratings). Due to the periodicity and symmetry of the structure, the analysis can be restricted to the half unit-cell problem depicted in Fig. 2(b). The TEM incident wave can be considered to propagate along a parallel-plate waveguide bounded by virtual electric walls, which can be modeled as a 


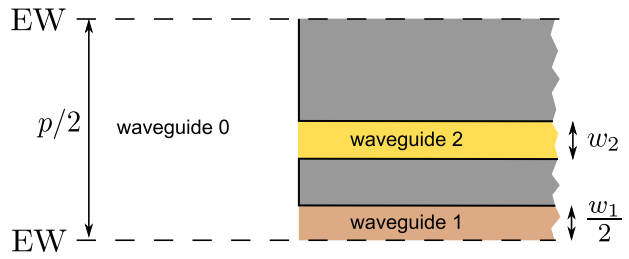

(a)

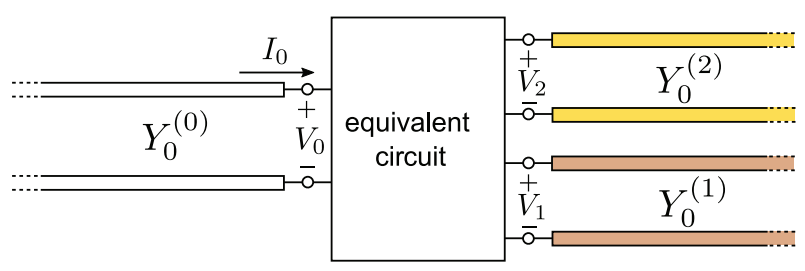

(b)

Fig. 3. (a) Generalized waveguide discontinuity problem associated with the half unit cell. (b) Circuit model of the discontinuity. The goal is to derive the topology of the equivalent circuit that models the discontinuity with analytical expressions for all of its parameters.

transmission line whose characteristic admittance ( $I$ to $V$ ratio) per unit length in the $x$-direction is given by

$$
Y_{0}^{(0)}=\frac{H_{\text {inc }}}{\frac{p}{2} E_{\text {inc }}}=\frac{2}{p \eta_{0}}
$$

with $\eta_{0}=\sqrt{\mu_{0} / \varepsilon_{0}}$ being the intrinsic impedance of free space. The two slits are also parallel-plate waveguides, which can similarly be modeled by two TEM transmission lines with characteristic admittances

$$
\begin{aligned}
& Y_{0}^{(1)}=\frac{2 \sqrt{\varepsilon_{\mathrm{r}}^{(1)}}}{w_{1} \eta_{0}} \\
& Y_{0}^{(2)}=\frac{\sqrt{\varepsilon_{\mathrm{r}}^{(2)}}}{w_{2} \eta_{0}} .
\end{aligned}
$$

Our first goal is to derive a circuit model for the discontinuity that couples the input line to the two output slit transmission lines, as illustrated in Fig. 3. This derivation is presented in Section II-A. Sections II-B and II-C then show the complete models for both the reflection and transmission compound gratings based on the previously derived discontinuity model. For simplicity, the structures are considered lossless in the previous analyses, and the effect of losses in the circuit parameters are introduced in Section II-D.

\section{A. Circuit Model for the Generalized Waveguide Discontinuity}

The tangential (to the screen) components of the fields at the left of the discontinuity $\left(z=0^{-}\right)$can be expanded in terms of the parallel-plate waveguide modes in the following way:

$$
\begin{aligned}
& E^{(0)}=\hat{\mathbf{y}} \cdot \mathbf{E}^{(0)}=1+R+\sum_{n=1}^{\infty} E_{n}^{(0)} \cos \left(k_{n} y\right) \\
& H^{(0)}=(-\hat{\mathbf{x}}) \cdot \mathbf{H}^{(0)}=(1-R) \xi_{0}^{(0)}-\sum_{n=1}^{\infty} \xi_{n}^{(0)} E_{n}^{(0)} \cos \left(k_{n} y\right)
\end{aligned}
$$

where all the terms have been normalized to the amplitude of the impinging wave, $E_{n}^{(0)}$ is the $n$th modal coefficient, $R$ represents the reflection coefficient for the impinging zeroth-order wave, and

$$
\begin{aligned}
k_{n} & =\frac{2 \pi n}{p} \\
\beta_{n}^{(0)} & =\sqrt{k_{0}^{2}-k_{n}^{2}} \\
\xi_{n}^{(0)} & =\frac{k_{0}}{\eta_{0} \beta_{n}^{(0)}}
\end{aligned}
$$

with $k_{n}$ being the $n$th mode cutoff wavenumber, $k_{0}$ the free-space wavenumber, $\beta_{n}^{(0)}$ is the wavenumber of the $n$th TM mode, and $\xi_{n}^{(0)}$ is its wave admittance. In particular,

$$
\xi_{0}^{(0)}=\frac{p}{2} Y_{0}^{(0)}
$$

Similarly, the fields in the slits at $z=0^{+}$could be represented using a modal expansion in terms of the corresponding parallel-plate waveguide modes. However, since the slits are narrow in comparison with the working wavelength, we only take into account the fundamental TEM mode, namely,

$$
\begin{aligned}
& \left.\begin{array}{l}
\mathbf{E}^{(1)}=E_{\mathrm{TEM}}^{(1)} \hat{\mathbf{y}} \\
\mathbf{H}^{(1)}=\xi^{(1)} E_{\mathrm{TEM}}^{(1)}(-\hat{\mathbf{x}})
\end{array}\right\}, \quad y \in\left[0, \frac{w_{1}}{2}\right] \\
& \left.\begin{array}{l}
\mathbf{E}^{(2)}=E_{\text {TEM }}^{(2)} \hat{\mathbf{y}} \\
\mathbf{H}^{(2)}=\xi^{(2)} E_{\text {TEM }}^{(2)}(-\hat{\mathbf{x}})
\end{array}\right\}, \quad y \in\left[h-\frac{w_{2}}{2}, h+\frac{w_{2}}{2}\right]
\end{aligned}
$$

where

$$
\xi^{(i)}=\frac{\sqrt{\varepsilon_{\mathrm{r}}^{(i)}}}{\eta_{0}}
$$

is the intrinsic admittance of the medium inside slit $i$. Note that

$$
\xi^{(1)}=\frac{w_{1}}{2} Y_{0}^{(1)} \quad \xi^{(2)}=w_{2} Y_{0}^{(2)} .
$$

To proceed with the derivation, the aperture field at the discontinuity plane $(z=0)$ is assumed to have the following form:

$$
\mathbf{E}_{\mathrm{a}}=\left[A(\omega) f_{1}(y)+B(\omega) f_{2}(y)\right] \hat{\mathbf{y}}
$$

where the frequency and the spatial dependence of the fields are factorized with $f_{i}(y)$ being the proposed field pattern at the $i$ th slit aperture. This implies that the spatial field profiles at the apertures are considered independent of frequency, although their complex amplitudes $A$ and $B$ may vary strongly with frequency. As will be discussed later, this approximation is very accurate for electrically narrow slits. Moreover, it can be checked that the effect of a slight variation of the aperture field profile does not have a significant effect on the transmission/reflection behavior of the structure due to the variational nature of the problem. In this work, the field at the apertures is assumed to be uniform,

$$
f_{i}(y)=1 \text { in slit } i, \quad f_{i}(y)=0 \text { elsewhere }
$$

which is consistent with the fact that only the fundamental TEM mode is considered inside the slits. Other aperture field profiles that took into account the correct edge behavior at the metal corners could have been employed, but, in our experience, the differences in the final results are almost negligible (as a general 
qualitative estimate of the limit of validity of this approximation, it has been found that the model provides reliable results for $w / \lambda \lesssim 0.3$ ). The $A$ and $B$ amplitudes of the field profiles can readily be related to the coefficients of the modal expansion by making use of standard Fourier analysis. Thus, projecting (4) and (14) over the modal profiles in the half unit-cell problem we have

$$
\begin{aligned}
\int_{0}^{p / 2} E^{(0)}(y) \cos \left(k_{m} y\right) \mathrm{d} y= & A \int_{0}^{w_{1} / 2} f_{1}(y) \cos \left(k_{m} y\right) \mathrm{d} y \\
& +B \int_{h-w_{2} / 2}^{h+w_{2} / 2} f_{2}(y) \cos \left(k_{m} y\right) \mathrm{d} y
\end{aligned}
$$

from which

$$
\begin{aligned}
\frac{p}{2}(1+R) & =\frac{w_{1}}{2} A+w_{2} B \\
\frac{p}{4} E_{n}^{(0)} & =\frac{w_{1}}{2} A \tilde{f}_{n}^{(1)}+w_{2} B \tilde{f}_{n}^{(2)} \cos \left(k_{n} h\right)
\end{aligned}
$$

where $\tilde{f}_{n}^{(i)}=\operatorname{sinc}\left(k_{n} w_{i} / 2\right)$ with $\operatorname{sinc}(x)=(\sin x) / x$. Also, from (10)-(14) it is clear that

$$
\begin{aligned}
& A=E_{\text {TEM }}^{(1)} \\
& B=E_{\text {TEM }}^{(2)}
\end{aligned}
$$

which allow us to rewrite (17) as

$$
V_{0}=V_{1}+V_{2}
$$

where $V_{0}$ is the voltage drop at the input port of the equivalent circuit in Fig. 3(b) and $V_{i}$ is the voltage drop at the output port corresponding to slit $i$.

The next step in the derivation is to impose the continuity of the power flux through the slit apertures. Due to the uniform electric field profile assumed at the apertures, it reduces to require the continuity of the magnetic field in weak (integral) form

$$
\begin{aligned}
\int_{0}^{w_{1} / 2} H^{(0)}(y) \mathrm{d} y & =\int_{0}^{w_{1} / 2} H^{(1)}(y) \mathrm{d} y \\
\int_{h-w_{2} / 2}^{h+w_{2} / 2} H^{(0)}(y) \mathrm{d} y & =\int_{h-w_{2} / 2}^{h+w_{2} / 2} H^{(2)}(y) \mathrm{d} y .
\end{aligned}
$$

Introducing (5) and (10) into (22) yields

$$
(1-R) \xi_{0}^{(0)}-\sum_{n=1}^{\infty} \xi_{n}^{(0)} E_{n}^{(0)} \tilde{f}_{n}^{(1)}=\xi^{(1)} E_{\mathrm{TEM}}^{(1)}
$$

Using (18), (9) and (13), this last equation can be rewritten as

$$
I_{0}=Y_{0}^{(1)} V_{1}+\bar{Y}_{11} V_{1}+\bar{Y}_{12} V_{2}
$$

with

$$
\begin{aligned}
& \bar{Y}_{11}=\frac{4}{p} \sum_{n=1}^{\infty} \xi_{n}^{(0)}\left[\tilde{f}_{n}^{(1)}\right]^{2} \\
& \bar{Y}_{12}=\frac{4}{p} \sum_{n=1}^{\infty} \xi_{n}^{(0)} \tilde{f}_{n}^{(1)} \tilde{f}_{n}^{(2)} \cos \left(k_{n} h\right)
\end{aligned}
$$

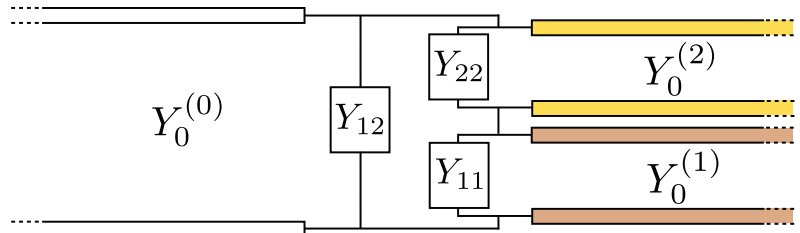

Fig. 4. Topology of the circuit model derived for the discontinuity that couples the impinging wave (input line) to the slit transmission lines.

and where the $I_{0}$ current, given by

$$
I_{0}=\frac{p}{2}(1-R) Y_{0}^{(0)}=\left(V_{0}^{+}-V_{0}^{-}\right) Y_{0}^{(0)}
$$

is the current flowing into the circuit at the input port. Similarly, using (23) we can obtain

$$
I_{0}=Y_{0}^{(2)} V_{2}+\bar{Y}_{12} V_{1}+\bar{Y}_{22} V_{2}
$$

with

$$
\bar{Y}_{22}=\frac{4}{p} \sum_{n=1}^{\infty} \xi_{n}^{(0)}\left[\tilde{f}_{n}^{(2)} \cos \left(k_{n} h\right)\right]^{2} .
$$

We can now use (21) to eliminate $V_{2}$ from (25), as well as $V_{1}$ from (29) to obtain

$$
\begin{aligned}
& I_{0}=Y_{12} V_{0}+\left[Y_{11}+Y_{0}^{(1)}\right] V_{1} \\
& I_{0}=Y_{12} V_{0}+\left[Y_{22}+Y_{0}^{(2)}\right] V_{2}
\end{aligned}
$$

where

$$
Y_{12}=\bar{Y}_{12} \quad Y_{i i}=\bar{Y}_{i i}-Y_{12} .
$$

A closer inspection of (31) and (32) already reveals the actual topology of the equivalent circuit. These equations tell us that the current flowing into the circuit follows two paths. One portion of the current flows through the $Y_{12}$ admittance with a total voltage drop $V_{0}$. The remaining current flows through the parallel connection of the $Y_{11}$ admittance and the transmission line corresponding to slit 1 (with voltage drop $V_{1}$ ), which is series connected to the parallel connection of the $Y_{22}$ admittance and the second slit line (voltage drop $V_{2}$ with $V_{1}+V_{2}=V_{0}$ ). The corresponding circuit topology is shown in Fig. 4. As a consistency check, (21), (25), and (29) can be combined to find the ratio $I_{0} / V_{0}$, which corresponds to the equivalent load admittance, $Y_{\text {eq }}$, met by the impinging wave. Thus, after some algebraic manipulations, $Y_{\text {eq }}$ can be expressed as

$$
Y_{\text {eq }}=Y_{12}+\left[\frac{1}{Y_{11}+Y_{0}^{(1)}}+\frac{1}{Y_{22}+Y_{0}^{(2)}}\right]^{-1}
$$

which is fully consistent with the circuit topology in Fig. 4. A similar derivation can be carried out for a compound grating having two different slits per period, and also for a structure with four slits per period distributed symmetrically in the unit cell. Clearly, the unit-cell and half unit-cell problems corresponding to these two latter structures are very similar to the case treated in detail above, with two output slit waveguides. Hence, the topology of their equivalent circuits would be the same as the 
one shown in Fig. 4 with only minor differences in the expressions of the circuit admittances, which are explicitly reported in Appendix A.

Although all the circuit parameters are obtained in analytical form, the expressions in (26), (27), and (30) involve infinite series, which is neither convenient from a computational point of view, nor to gain physical insight from the model. Following the guidelines proposed in [16], this drawback can be overcome by considering separately the contribution of the lowand high-order modes to the circuit parameters, as explained next. For evanescent modes (high-order modes), the modal admittances in (8) are imaginary and can be written as

$$
\xi_{n}^{(0)}=\mathrm{j} \omega \frac{C_{n}}{\sqrt{1-\left(\frac{\omega}{\omega_{n}}\right)^{2}}} \quad C_{n}=\frac{\varepsilon_{0}}{k_{n}}
$$

where $\omega_{n}=c k_{n}$ is the cutoff angular frequency of the $n$th mode ( $c$ is the speed of light in free space). For those modes whose cutoff frequency is significantly above the frequency range of interest, it is found that $\left(\omega / \omega_{n}\right)^{2} \ll 1$ and, therefore, their contribution to the equivalent circuit admittances can be well approximated as a frequency-independent capacitance $\left(\xi_{n}^{(0)} \approx\right.$ $\left.\mathrm{j} \omega C_{n}\right)$. Assuming that this approximation is valid for modes with order higher than a given $N$, the admittances in (26), (27), and (30) can thus be split into the following two contributions:

$$
\bar{Y}_{i j}=\bar{Y}_{i j}^{(\mathrm{lo})}+\mathrm{j} \omega \bar{C}_{i j}
$$

where the low-order (lo) admittances represent the dynamical (i.e., with the complete nonlinear frequency dependence) contribution of the first $N$ modes to the infinite series, and the capacitances $\bar{C}_{i j}$ are given by

$$
\begin{aligned}
& \bar{C}_{11}=\frac{4}{p} \sum_{n=N+1}^{\infty} C_{n}\left[\tilde{f}_{n}^{(1)}\right]^{2} \\
& \bar{C}_{22}=\frac{4}{p} \sum_{n=N+1}^{\infty} C_{n}\left[\tilde{f}_{n}^{(2)} \cos \left(k_{n} h\right)\right]^{2} \\
& \bar{C}_{12}=\frac{4}{p} \sum_{n=N+1}^{\infty} C_{n} \tilde{f}_{n}^{(1)} \tilde{f}_{n}^{(2)} \cos \left(k_{n} h\right) .
\end{aligned}
$$

According to (33), it is apparent that this decomposition translates directly to the admittances in the model, namely,

$$
Y_{i j}=Y_{i j}^{(\mathrm{lo})}+\mathrm{j} \omega C_{i j}
$$

with

$$
C_{i i}=\bar{C}_{i i}-\bar{C}_{12} \quad C_{12}=\bar{C}_{12}
$$

and a similar relation for the $Y^{(\mathrm{lo})}$ dynamical admittances. The resulting equivalent circuit is represented in Fig. 5.

In regards to the value of $N$, our experience says that $N$ can often be chosen as the number of high-order propagative modes at the highest frequency of interest. However, as a general criterion for the systematic application of the model, it is advised to incorporate also the first evanescent mode into the low-order dynamical admittances. According to this general criterion, we will take $N=1$ when working below the onset of the first

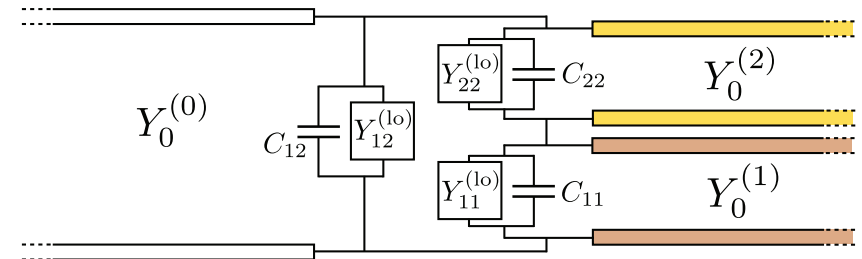

Fig. 5. Circuit model in Fig. 4, but now showing the separate contribution of the low-order (lo), i.e., propagative or close to cutoff, modes and the frequency-independent capacitances that incorporate the global contribution of all the remaining high-order evanescent modes.

higher order mode (nondiffraction regime), $N=2$ for frequencies within the first diffraction order regime (the first higher order mode is propagative, but the second is still evanescent), and so on. It is important to recall that the values of the capacitances involved in the proposed model are independent of frequency. Therefore, when performing a frequency sweep in order to obtain the transmission/reflection spectrum of the structure in a given frequency range, the computation of the infinite series is carried out only once. If desired, the numerical series can be quickly computed using the methods reported, for instance, in [29, App. A]. The above considerations certainly makes the proposed dynamical equivalent circuit very efficient from a computational point of view.

Here it is interesting to point out that, for frequencies well below the onset of the first grating lobe, all the higher order modes can actually be incorporated into the capacitances (which is equivalent to take $N=0$ ). It leads to an equivalent circuit with all its lumped parameters being frequency-independent capacitances. In this way we recover the quasi-static capacitive network proposed in [14] by some of the authors. However, it should be noted that [14] did not provide any derivation of the circuit model, but rather a heuristic proposal where the values of the capacitances had to be extracted from an external quasi-static and relatively complex numerical calculation. In contrast, a rigorous derivation is now given, which results in analytical expressions for all the circuit parameters. Moreover, the model derived here is easily applicable to high frequencies well beyond the diffraction limit thanks to the fact that the expressions found for the circuit parameters incorporate analytically the dynamical frequency dependence of the higher order modes as they approach (and eventually exceed) their cutoff frequencies. When working within the diffraction regime, the modal admittances of the propagative higher order modes is real and can be written as

$$
\xi_{n}^{(0)}=\frac{1}{\eta_{0}} \frac{\omega}{\sqrt{\omega^{2}-\omega_{n}^{2}}} .
$$

In consequence, the contributions of each propagative higher order mode to the admittances in the equivalent circuit are frequency-dependent resistances, and the power dissipated in these resistances can be interpreted as the power transferred to the corresponding grating lobe. When the operation frequency is below the onset of the first grating lobe, although not far from this limit, all the lumped admittances are purely imaginary, but the equivalent capacitors exhibit a frequency dependence that is essential to explain extraordinary transmission effects. The use of purely 


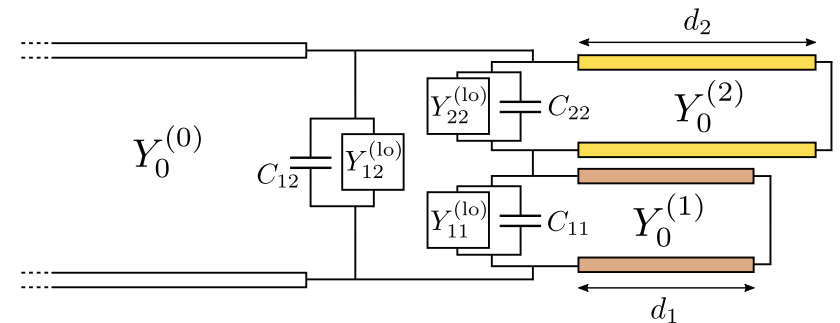

Fig. 6. Complete circuit model for a reflection compound grating such as that in Fig. 2.

quasi-static values for those capacitors [14] cannot accurately account for the behavior of the compound grating at frequencies close to the first RW anomaly.

\section{B. Equivalent-Circuit Model for Reflection Structures}

Once the equivalent circuit of the discontinuity that couples the impinging wave to the slits is known, the complete models for a reflection structure as that sketched in Fig. 2 can be obtained quite straightforwardly after incorporating the termination of the slits into the model. This is achieved by simply placing a short-circuit termination at the corresponding distance from the discontinuity in each slit transmission line, as shown in Fig. 6. The equivalent load admittance met by the impinging wave is then given by

$$
Y_{\mathrm{eq}}=Y_{12}+\left[\frac{1}{Y_{11}+Y_{\mathrm{in}}^{(1)}}+\frac{1}{Y_{22}+Y_{\mathrm{in}}^{(2)}}\right]^{-1}
$$

where $Y_{\text {in }}^{(i)}$ is the input admittance to the short-circuited length of transmission line corresponding to slit $i$; namely,

$$
Y_{\mathrm{in}}^{(i)}=-\mathrm{j} Y_{0}^{(i)} \cot \left(\beta_{0}^{(i)} d_{i}\right)
$$

with

$$
\beta_{0}^{(i)}=k_{0} \sqrt{\varepsilon_{\mathrm{r}}^{(i)}}
$$

being the wavenumber of the $i$ th slit TEM mode. The reflection coefficient is finally obtained using standard transmission-line theory as

$$
R=\frac{Y_{0}^{(0)}-Y_{\mathrm{eq}}}{Y_{0}^{(0)}+Y_{\mathrm{eq}}} .
$$

At this point, it is worth mentioning that the previous derivations have been carried out assuming normal incidence. The consideration of oblique incidence has some relevant consequences. Even though a similar analytical description with compact and simple expressions for the involved admittances can be derived, it turns out that a circuit topology like that in Fig. 4 could not account for the obtained linear relations between the relevant electrical quantities. Another equivalent circuit could still be drawn if voltage-controlled current sources were used (the authors have verified that this model for oblique incidence fulfills fundamental requirements such as power conservation and reciprocity). However, the physical

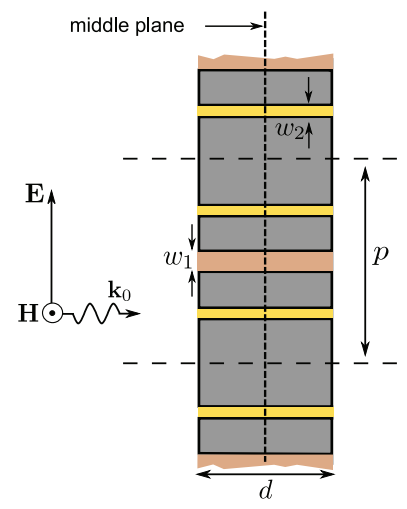

Fig. 7. Sketch of a transmission compound grating with three slits per period distributed symmetrically in the unit cell. The structure is symmetric with respect to the vertical middle plane.

interpretation of that model is not straightforward and it will not be considered in this paper.

\section{Equivalent-Circuit Model for Transmission Structures}

For the case of a transmission grating such as that sketched in Fig. 7, it is possible to take advantage of the symmetry of the structure with respect to the vertical middle plane of the screen to decompose the problem into even- and odd-excitation (with respect to the middle plane) half problems. For the even excitation case, the middle plane behaves as a virtual magnetic wall. This magnetic-wall condition at the middle of the screen translates into open-circuit terminations of the slit transmission lines at a distance $d / 2$ from the discontinuity ( $d$ is the screen thickness). For the odd excitation case, the middle plane behaves as a virtual electric wall corresponding to short-circuit terminations. Taking into account these considerations, the circuit models for the even- and odd-excitation half problems are depicted in Fig. 8. The equivalent load admittance for the even/odd excitation circuits, $Y_{\mathrm{eq}}^{(\mathrm{e} / \mathrm{o})}$, is thus given by (43), after replacing the input admittances with the following ones corresponding to the circuits in Fig. 8:

$$
Y_{\mathrm{in}}^{(i)}=\mathrm{j} Y_{0}^{(i)} \times \begin{cases}\tan \left(\frac{\beta_{0}^{(i)} d}{2}\right) & \text { even excitation } \\ -\cot \left(\frac{\beta_{0}^{(i)} d}{2}\right) & \text { odd excitation. }\end{cases}
$$

The reflection coefficients for the even/odd excitations half problems are then computed from

$$
S_{11}^{(\mathrm{e} / \mathrm{o})}=\frac{Y_{0}^{(0)}-Y_{\mathrm{eq}}^{(\mathrm{e} / \mathrm{o})}}{Y_{0}^{(0)}+Y_{\mathrm{eq}}^{(\mathrm{e} / \mathrm{o})}} .
$$

Finally, the reflection and transmission coefficients for the complete structure are obtained as

$$
\begin{aligned}
R & =\frac{1}{2}\left(S_{11}^{(\mathrm{e})}+S_{11}^{(\mathrm{o})}\right) \\
T & =\frac{1}{2}\left(S_{11}^{(\mathrm{e})}-S_{11}^{(\mathrm{o})}\right) .
\end{aligned}
$$

\section{Introduction of Losses}

Material losses are separately considered (as usual) as dielectric losses in the media filling the slits and ohmic losses in 


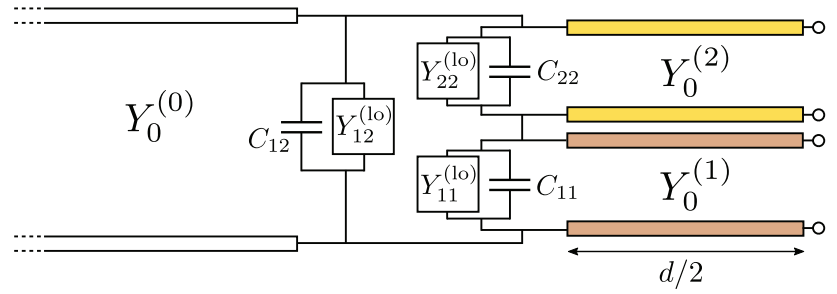

(a)

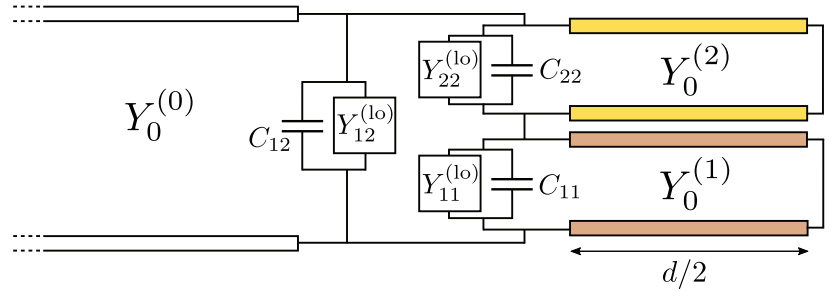

(b)

Fig. 8. Circuit models for the: (a) even and (b) odd excitation of the transmission structure in Fig. 7. The models differ in the open/short-circuit terminations of the slit transmission lines at a distance $d / 2$, which correspond to the middle plane behaving as a virtual magnetic/electric wall.

the metallic screen. The dielectric losses are accounted for by simply taking their corresponding complex-valued permittivities

$$
\hat{\varepsilon}_{\mathrm{r}}^{(i)}=\varepsilon_{\mathrm{r}}^{(i)}\left(1-\mathrm{j} \tan \delta_{i}\right)
$$

where $\tan \delta_{i}$ is the dielectric loss tangent of the medium inside slit $i$. From a practical point of view, dielectric losses are rigorously incorporated in the model by introducing the complex permittivities into the expressions of the slit characteristic admittances (2) and (3), and wavenumbers (45).

Although high-conductivity metals behave almost as perfect conductors at microwave frequencies, ohmic losses may be significant at the frequencies of interest when resonances appear. In order to incorporate ohmic losses in the present circuit model, the basic ideas in [33] can similarly be applied here. In brief, the slits are now considered lossy parallel-plate waveguides with complex wavenumbers, which, under the good conductor approximation and strong skin effect conditions, are given by

$$
\hat{\beta}_{0}^{(i)}=\beta_{0}^{(i)} \sqrt{1+\frac{(1-\mathrm{j}) \delta_{\mathrm{s}}}{w_{i}}}
$$

where $\beta_{0}^{(i)}$ is the wavenumber for the lossless structure and $\delta_{\mathrm{s}}=$ $\sqrt{2 / \omega \mu_{0} \sigma}$ is the skin penetration depth into the metallic walls ( $\sigma$ is the screen conductivity). The characteristic admittances of the slit transmission lines are then given by

$$
\hat{Y}_{0}^{(1)}=\frac{2 \hat{\xi}^{(1)}}{w_{1}} \quad \hat{Y}_{0}^{(2)}=\frac{\hat{\xi}^{(2)}}{w_{2}}
$$

with

$$
\hat{\xi}^{(i)}=\frac{\varepsilon_{\mathrm{r}}^{(i)} k_{0}}{\eta_{0} \hat{\beta}_{0}^{(i)}}
$$

being the complex value of the wave admittance of the fundamental mode in slit $i$. For a transmission grating, ohmic losses

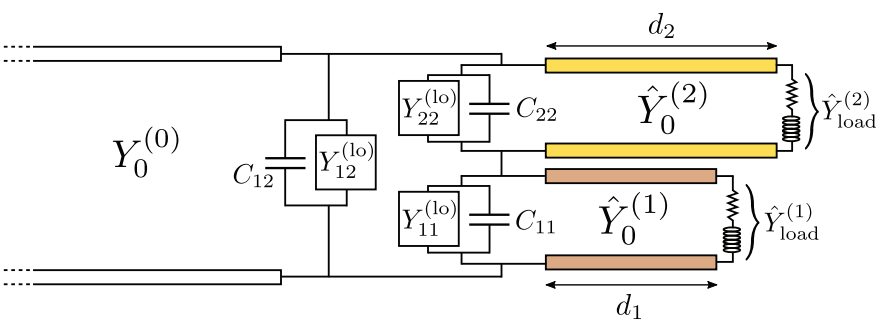

Fig. 9. Sketch of the circuit model for a lossy reflection grating, showing the complex load admittances at the termination of the slit lines.

are introduced in the model just by replacing $Y_{0}^{(i)}$ and $\beta_{0}^{(i)}$ with their corresponding complex values $\hat{Y}_{0}^{(i)}$ and $\hat{\beta}_{0}^{(i)}$ in (47). In the case of a reflection grating, the losses at the bottom wall of the slits can also be accounted for by terminating the slit transmission line with imperfect short loads, as sketched in Fig. 9. Including these loads is important because high current density levels can appear in the short-circuit termination of the slits at resonance. Under strong skin effect, the values of the load admittances are given by

$$
\hat{Y}_{\text {load }}^{(1)}=\frac{2}{w_{1} Z_{\mathrm{s}}} \quad \hat{Y}_{\text {load }}^{(2)}=\frac{1}{w_{2} Z_{\mathrm{s}}}
$$

where

$$
Z_{\mathrm{s}}=\frac{1+\mathrm{j}}{\sigma \delta_{\mathrm{s}}}
$$

is the surface impedance of the metal. In consequence, the input admittances in (44) have to be replaced with

$$
Y_{\text {in }}^{(i)}=\hat{Y}_{0}^{(i)} \frac{\hat{Y}_{\text {load }}^{(i)}+\mathrm{j} \hat{Y}_{0}^{(i)} \tan \left(\hat{\beta}_{0}^{(i)} d_{i}\right)}{\hat{Y}_{0}^{(i)}+\mathrm{j} \hat{Y}_{\text {load }}^{(i)} \tan \left(\hat{\beta}_{0}^{(i)} d_{i}\right)} .
$$

It should be pointed out that the above considerations take into account ohmic losses in all the slit walls, but not in the metallic surfaces out of the slits. This effect could be approximately introduced by adding appropriate resistors. However, as already mentioned above, this contribution can be neglected for good conductors (e.g., metals at microwave frequencies) since ohmic losses are significant only under specific resonance conditions where most of the power is dissipated inside the slits.

As a final comment it should be noted that, even though the analytical developments in this paper have been carried out for the case of three slits per period, the procedure can easily be extended to more complex structures having a higher number of slits per period. Details for a five (or six) slits per cell are given in Appendix B. Once again, an analytical circuit is obtained, although with a more cumbersome topology. Also, one example involving a structure with five slits per period has been included in the results section together with the sketch of the associated equivalent circuit in Appendix B.

\section{Numerical AND ANAlytical Results}

This section presents some results to check the validity and accuracy of the proposed dynamical model for compound gratings as well as the advantages of this model over the previously reported quasi-static version [14]. 


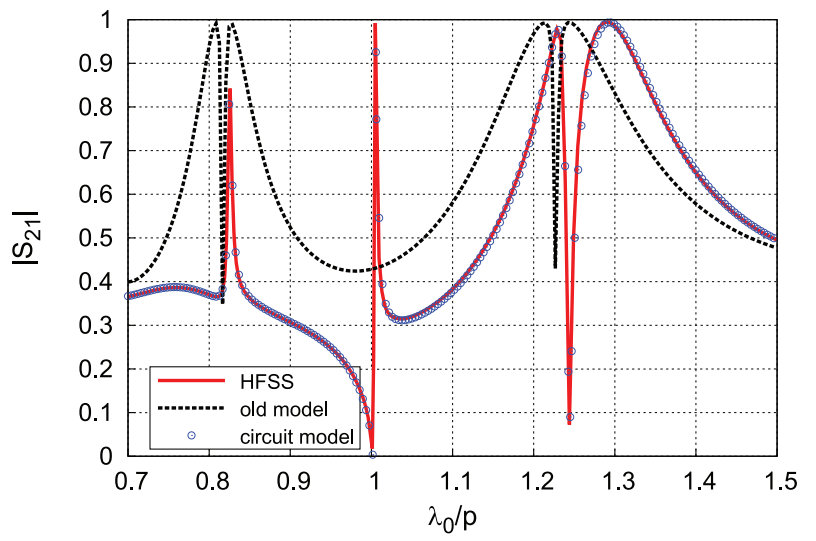

Fig. 10. Comparison of the transmission coefficients (magnitude) computed with HFSS (red solid line) with the quasi-static [14] (old model) approximation (black dashed line) and with the dynamical circuit proposed in this paper (blue circles). The considered structure is the three slits per period compound grating analyzed in [14, Fig. 5]. Dimensions: period, $p=10 \mathrm{~mm}$; metal slab thickness, $d=11.4 \mathrm{~mm}, w_{1}=w_{2}=0.8 \mathrm{~mm}$, and separation between the centers of the slits of $1.6 \mathrm{~mm}$.

\section{A. Compound Transmission Gratings}

As a first example, the transmission-like compound gratings analyzed in [14, Fig. 5] are considered. In that paper it was shown that the quasi-static model was suitable to explain the existence of narrow transmission dips inside the FP transmission bands for compound gratings based on groups of three or more slits per period (normal incidence case). This phenomenon is closely related to the so-called classical electromagnetically induced transparency. In [14, Fig. 5], it could be seen that the quantitative agreement between the heuristic circuit model predictions and full-wave simulations (HFSS) was quite good for the FP resonance occurring at frequencies for which $\lambda_{0} / p \approx 2.5$ where $\lambda_{0}$ is the free-space wavelength. However, the quantitative agreement significantly deteriorates as the operation frequency approaches the onset of the diffraction regime $\left(\lambda_{0} / p \lesssim 1\right)$. A detailed exploration of this frequency range for the three slits per period structure in [14, Fig. 5] is now shown in Fig. 10. In this figure, numerical (HFSS) results are compared with the predictions of the quasi-static model [14] (old model) and with the dynamical model introduced in this paper. From the figure it is clear that the quasi-static model still predicts the existence of the transmission dip in the middle of the FP resonance $\left(\lambda_{0} / p \approx 1.23\right)$, but its quantitative accuracy is very poor. In contrast, the results obtained with the dynamical model are indistinguishable from numerical data. This good matching is caused by the incorporation of the frequency-dependent behavior of the capacitances in the model, which is quite relevant in that frequency region. These capacitances exhibit a singular behavior around the RW frequency $\left(\lambda_{0} / d=1\right)$, in such a way that an extraordinary transmission peak followed by a Rayleigh transmission zero (Fano-like resonance) is predicted. This fact is in perfect agreement with the numerical calculation (see region around $\lambda_{o} / p=1$ in Fig. 10), but it is completely lost by the quasi-static model. The results provided by the quasi-static model above the diffraction threshold $\left(\lambda_{o} / p<1\right)$ are completely meaningless, while the data computed with the new dynamical model perfectly match the HFSS results within the diffraction region $(\lambda \leq p)$. The above results then show that the dynamical circuit model is advantageous when used at relatively high frequencies to characterize transmission-like compound gratings, and it is indispensable for frequencies around and above the first RW anomaly. In particular, the quasi-static model cannot account for the existence of RW anomalies, as well as the extraordinary transmission phenomenon. Actually, the quasi-static model for compound structures [14] is useful only for the modeling of FP-like resonances. Note that the nature of extraordinary transmission peaks is completely different, being related to the periodicity of the structure rather than the thickness of the metal slab [6], [19].

\section{B. Compound Reflection Gratings}

The case of reflection gratings is of special interest in the context of this paper because their most interesting properties appear in the diffraction regime. For lossless structures within the sub-diffraction regime, nothing relevant is expected in the magnitude of the reflection coefficient although its phase experiences fast variations around certain frequency points. In this sub-diffraction regime, the proposed dynamical model substitutes the static capacitors of the heuristic model [14] by other suitable capacitors whose static capacitances are not the ones given by the corresponding solution of Laplace's equation. The new static capacitors account for the capacitive effects linked to high (enough)-order evanescent TM modes. If the operation frequency is sufficiently close to the onset of any high-order mode, the corresponding previously taken static capacitor should now incorporate a frequency-dependent contribution associated with the involved TM mode. This contribution is singular at cutoff and explains, in the frame of the circuit model, the observation of perfect specular reflection at the RW anomaly frequency points. (Specular reflection is the reflection corresponding to the zeroth-order impinging mode according to the terminology of the equivalent waveguide problem used in this paper.) Note that RW anomalies of the grating correspond to the onset of TM modes in the waveguide model. Thus, perfect specular reflection at RW anomalies is trivially explained, in the frame of the circuit model, by the singular behavior of the frequencydependent capacitors. Moreover, the model also accounts for the transfer of power to the successive diffraction orders by means of frequency-dependent conductances (resistances) that are shunt connected to the capacitors. For lossless conductors and below the onset of the first grating lobe, it is obvious that the magnitude of the specular reflection coefficient must be unity. However, above the first RW frequency, the specular reflection coefficient drastically drops because a significant part of the impinging power is transferred to nonspecular grating lobes. This is what happens with simple gratings (one slit per period) in all the frequency span between any two successive RW points. However, in the case of compound gratings, narrow peaks of perfect specular reflection have been reported at some frequency points between RW anomalies [38], [39]. These peaks were attributed to the existence of phase resonance phenomena. Our circuit model can also provide a simple explanation to this effect. In the case of a lossless system involving three slits per period, the pertinent equivalent circuit is the one in Fig. 6. The 


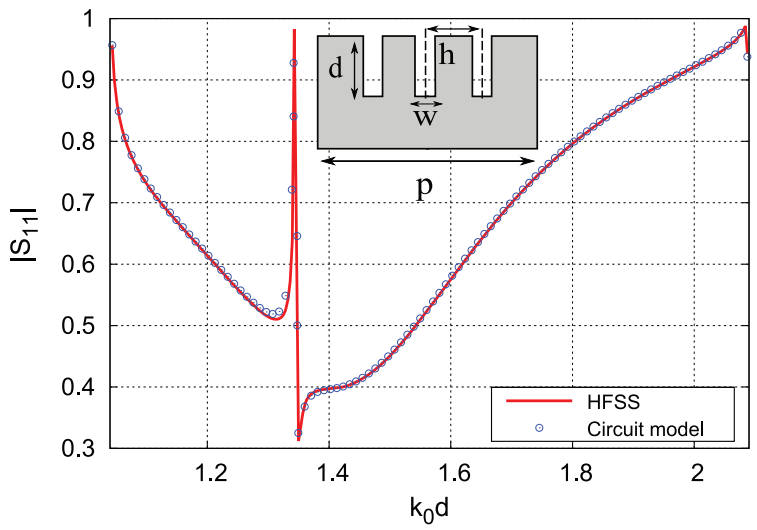

(a)

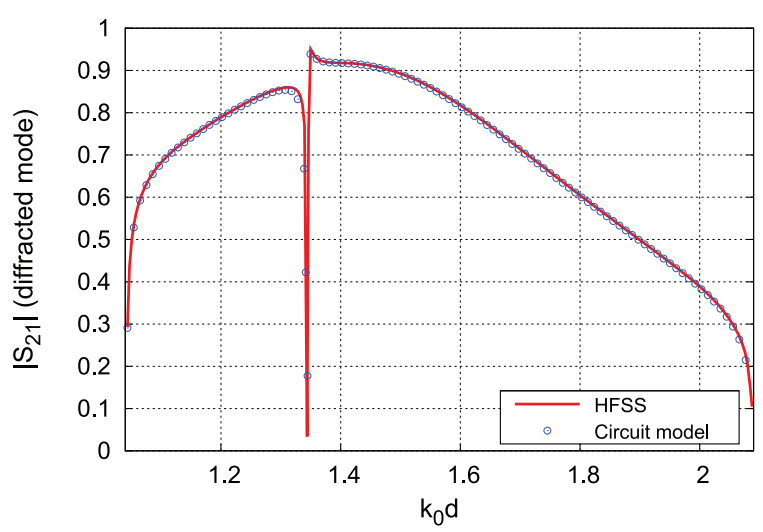

(b)

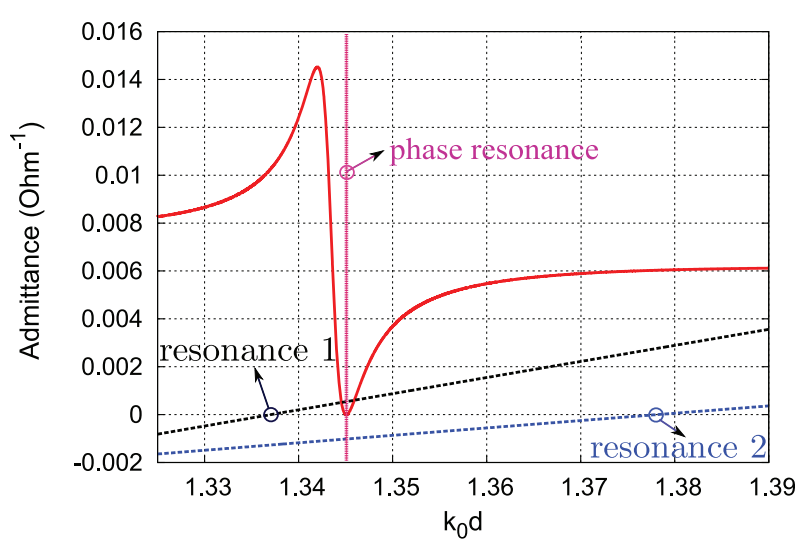

(c)

Fig. 11. (a) Magnitude of $S_{11}$ (specular efficiency) for the three slits per period compound reflection grating considered in [38, Fig. 2(b)] (see inset for unit cell). Normalized dimensions: $p / d=6.0, w / d=0.30, h / d=0.50$. (b) Transmission to the first diffraction order (magnitude). (c) Dashed lines: imaginary parts of the admittances associated with the resonant circuits involved in the modeling of the two independent slits. Solid line: real part of the overall equivalent admittance loading the input transmission line [see (43)].

results provided by HFSS and our dynamical circuit model for the specular efficiency pattern of the three-slits per period reflection compound grating analyzed in [38, Fig. 2(b)] are shown in Fig. 11(a). The transmission from the impinging wave to the first diffraction order is plot in Fig. 11(b). Normalized frequency is used as in [38]. Very good agreement between analytical and numerical data can be observed. Similar good agreement has been verified with the curve reported in [38, Fig. 2(b)], which was obtained using a mode-matching scheme. Note that a perfect specular reflection peak appears at about $k_{0} d=1.35$. This peak is associated with the existence of a zero of the real part of the equivalent admittance [see (43)] loading the transmission line that represents the impinging uniform plane wave. This zero always appears in the range of frequencies defined by the resonances of the individual resonators composed by the short-circuited slits with their associated external edge capacitances. These resonances are marked in Fig. 11(c) with circles and correspond to the points where the imaginary parts of the overall admittances (dashed lines) associated with slits 1 and 2 are null. The real part of (43) is represented in the same figure as a solid line. It can be observed that this quantity vanishes at around $k_{0} d=1.35$. Therefore, at certain frequency point, all the impinging power is specularly reflected and no transfer of power to grating lobes is allowed, as it was numerically predicted in [38]. Our circuit model provides an analytical explanation for this fact.

\section{Ohmic Losses}

In the previous examples, perfect electric conductors have been assumed. However, our model can also deal with lossy materials (metal and dielectric). Fig. 12 shows the magnitude and phase of the reflection coefficient of the structure studied in Fig. 11, but including metal losses (aluminum is considered in this example). Note that normalized frequency values should not be used in this case. A specific choice has been done for the dimensions (maintaining their relative values) and thus a specific frequency range must be explored. It can be appreciated that circuit-model and numerical (HFSS) results agree very well over the whole analyzed frequency band. As can be observed in Fig. 12(a), the specular reflection peak is greatly affected by metal losses (the magnitude of $S_{11}$ is now about 0.84 instead of 1). A small transmission dip in the magnitude of $S_{11}$ can also be recognized close to the onset of the diffraction regime. This dip is related to both phase resonance and extraordinary transmission and it is again accurately reproduced with our dynamical circuit model. In this example the phase of the reflection coefficient has also been plotted in Fig. 12(b) to show the good performance of our model even for phase calculations.

\section{Structures With Five Slits Per Period}

Although the analytical details in Section II have been presented for the simplest case exhibiting exotic behavior under normal incidence conditions (three symmetrically placed slits per period), the procedure employed in this paper can easily be extended to any number of slits. Thus, the analytical model described in Appendix B has been used to generate the data reported in [39, Fig. 2(a)] (five slits per period). These data are shown in Fig. 13(a) (note that normalized frequencies are used, as in [39]). Agreement with full-wave computations is very good and the two expected specular reflection points are reproduced very accurately. The reason for the existence of those points can be appreciated in Fig. 13(b), where two zeros of the real part of the loading equivalent admittance can be clearly noticed. Note that those zeros are again around the resonance region of the three slits of the irreducible unit cell. The equivalent 


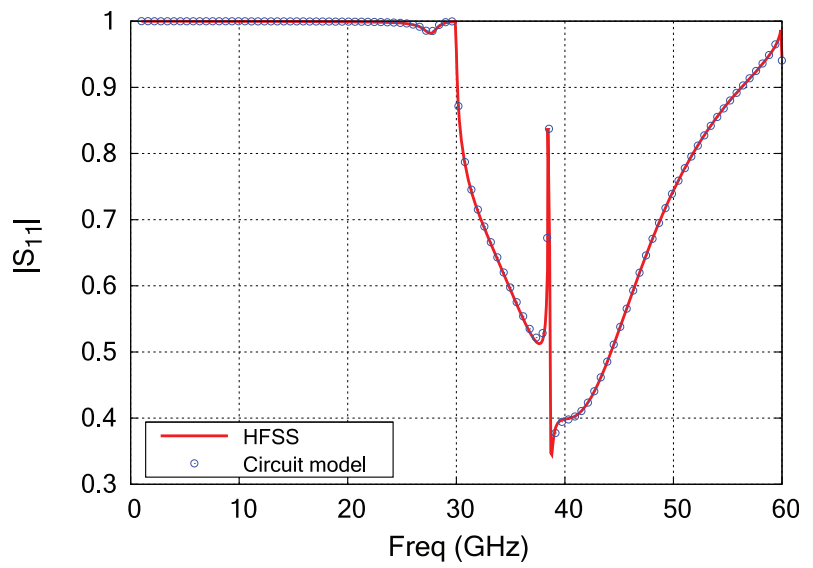

(a)

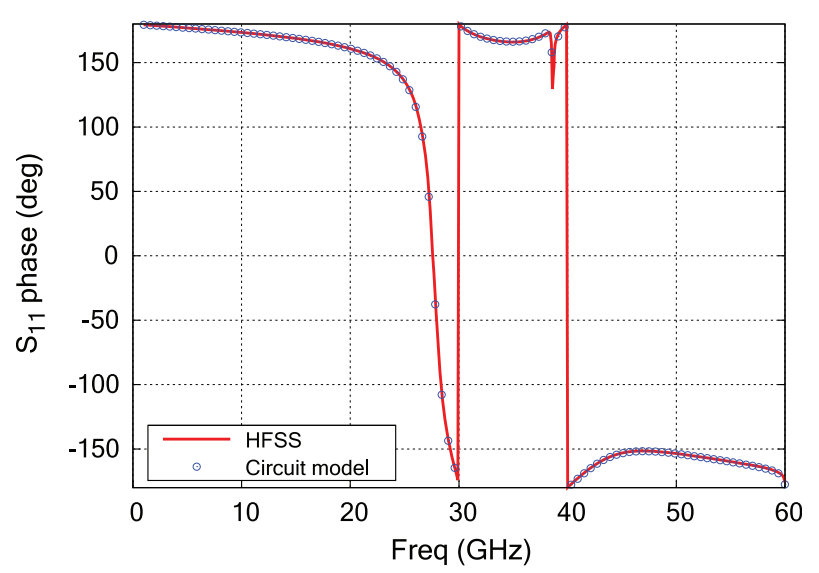

(b)

Fig. 12. (a) Magnitude of the reflection coefficient versus frequency. (b) Phase of the reflection coefficient versus frequency. Structure parameters: $p=10 \mathrm{~mm}, w_{1}=w_{2}=0.8 \mathrm{~mm}, d_{1}=5.641 \mathrm{~mm}, d_{2}=5.7 \mathrm{~mm}$, $h=1.6 \mathrm{~mm}, \varepsilon_{\mathrm{r}}^{(1)}=\varepsilon_{\mathrm{r}}^{(2)}=1, \sigma(\mathrm{Al})=3.8 \times 10^{7} \mathrm{~S} / \mathrm{m}$.

circuit allows us to predict again the existence of such zeros and the perfect specular reflection peaks.

\section{E. Comparison With Experimental Results}

As a final example, the compound grating with experimental data reported in [42] is considered in Fig. 14. This figure clearly shows that our model matches the measured transmission coefficients very accurately. For the given values of the groove depth, the two explored frequency regions plotted in Fig. 14(a) and (b) correspond to FP-like resonances, which could have been reasonably reproduced with the quasi-static model reported in [14]. Nevertheless, the dynamical-circuit results shown in these figures show a better quantitative matching with the additional advantage that the static capacitances used in the model have been generated using analytical expressions rather than running a finite-element Laplace's solver [14].

Although all the examples reported in this section have involved air-filled slits, many other structures with dielectric-filled slits have also been simulated with HFSS and compared with our proposed model showing similar good agreement. These examples have not been included to save space and only examples showing interesting phenomena have been shown. To have an idea about the computational savings

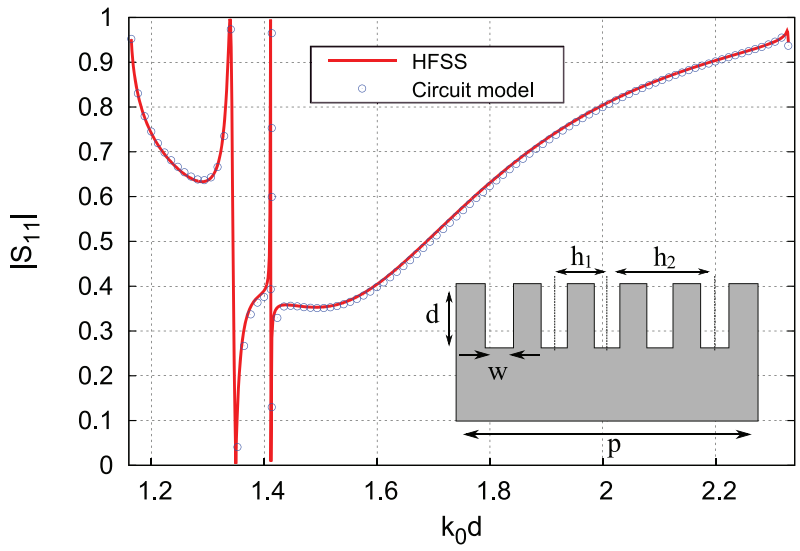

(a)

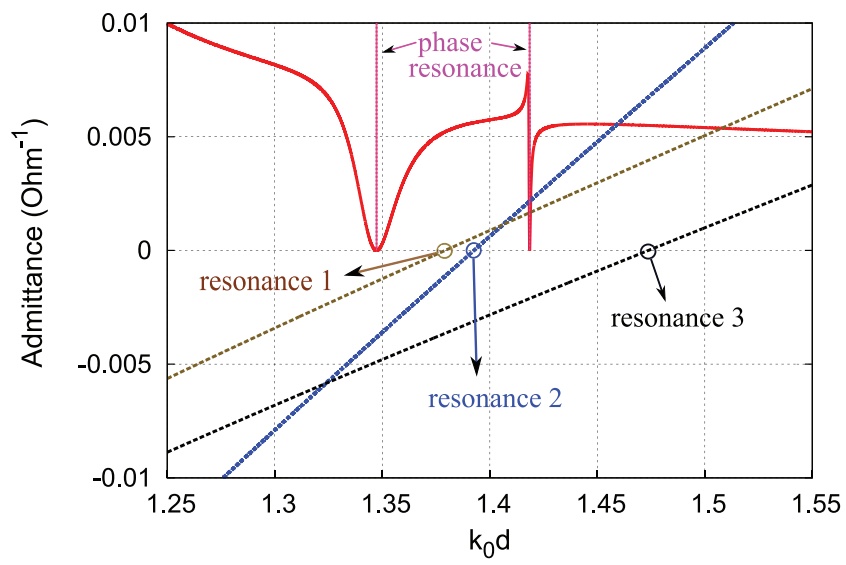

(b)

Fig. 13. Results obtained for a compound grating with five identical slits per period. (a) Magnitude of the reflection coefficient and (b) imaginary parts (dashed lines) of the admittances of the individual independent slits and real part (solid line) of the admittance loading the input transmission line. Dimensions: $p=10.0 \mathrm{~mm}, w_{1}=w_{2}=w_{3}=0.37 \mathrm{~mm}, d_{1}=d_{2}=d_{3}=1.852 \mathrm{~mm}$, $h_{2}=2 h_{1}=1.37 \mathrm{~mm}$.

achieved with the use of the analytical approach, it must be said that HFSS computations required from several minutes to a couple of hours, depending on the complexity of the structure and the number of points employed to draw a given curve. The analytical CPU times were always below 1s in the same computer platform.

\section{CONCLUSION}

An equivalent-circuit model has been rigorously deduced for compound gratings with an arbitrary number of slits per unit cell when illuminated with a TM-polarized normally impinging uniform plane wave. The equivalent circuit incorporates dynamical features that were absent in previous circuit models reported in the literature. All the parameters of the newly proposed circuit model can easily be computed using analytical expressions in such a way that a very low numerical effort is required. Moreover, the equivalent circuit provides an alternative easy way to understand the complex behavior of compound gratings working both in transmission and in reflection. Material losses have also been added to the model in a straightforward way. Analytical data agree very well with HFSS simulations and with previously reported measured results. 


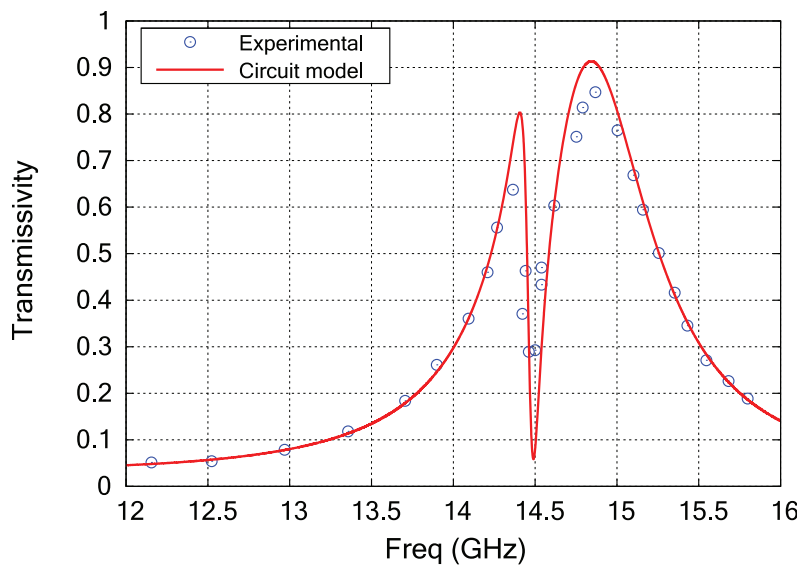

(a)

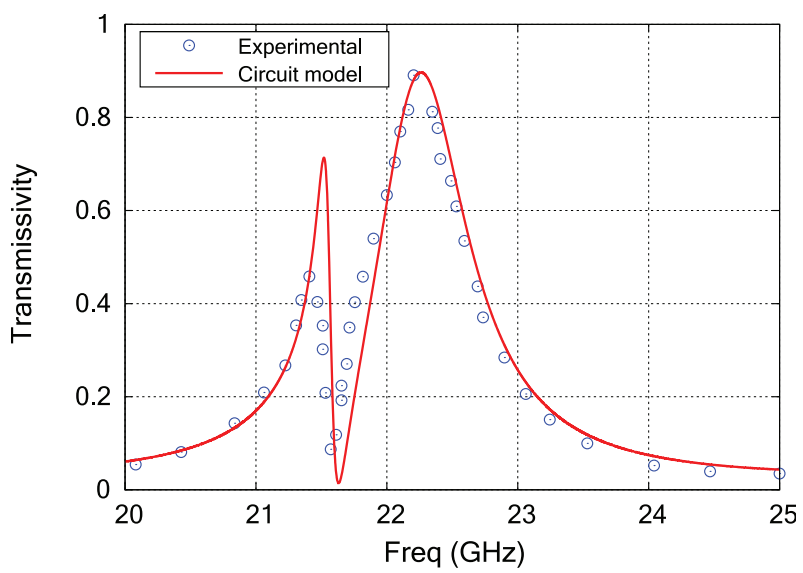

(b)

Fig. 14. Transmissivitty versus frequency for two different FP bands of the compound grating experimentally studied in [42]. The red solid line corresponds to the analytical results and the circles are samples of the experimental results in [42]. Structure parameters: $p=10 \mathrm{~mm}, w_{1}=w_{2}=0.5 \mathrm{~mm}, d_{1}=d_{2}=$ $9.9 \mathrm{~mm}, h=3.375 \mathrm{~mm}, \varepsilon_{\mathrm{r}}^{(1)}=\varepsilon_{\mathrm{r}}^{(2)}=1, \sigma=3.816 \cdot 10^{7} \mathrm{~S} / \mathrm{m}$.

\section{APPENDIX A}

The half unit-cell problem for a structure with four slits symmetrically distributed within the unit cell is shown in Fig. 15(a), whereas Fig. 15(b) shows the unit cell for a structure with two different slits per period. In both cases, there are two slits in the irreducible (half) unit-cell problem. Thus, the topology of the circuit model for the discontinuity is the same as shown in Fig. 4. The specific expressions of the circuit parameters for either case are given next. In both cases, the characteristic admittances of the transmission lines associated with the slits are

$$
Y_{0}^{(i)}=\frac{\sqrt{\varepsilon_{\mathrm{r}}^{(i)}}}{w_{i} \eta_{0}}
$$

The input line admittance is the same as in (1) for the symmetrical problem, whereas, for the nonsymmetrical case, it does not include the factor 2; namely,

$$
Y_{0}^{(0)}=\frac{1}{p \eta_{0}}
$$

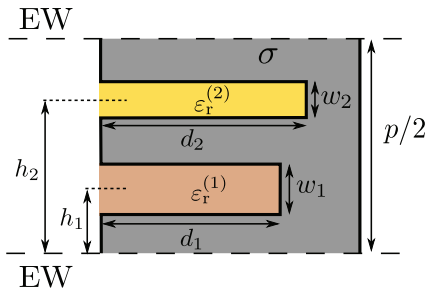

(a)

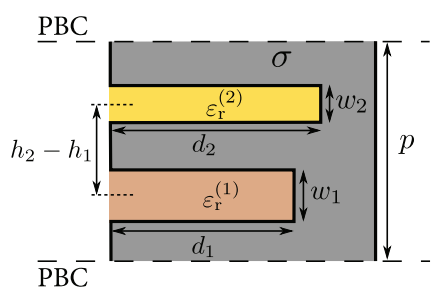

(b)
Fig. 15. (a) Half unit-cell problem for a reflection grating with four slits per period, distributed symmetrically within the unit cell. (b) Unit cell with two different slits. Due to the absence of symmetry, in this case the boundary walls of the cell are not virtual electric walls, but periodic boundary conditions.

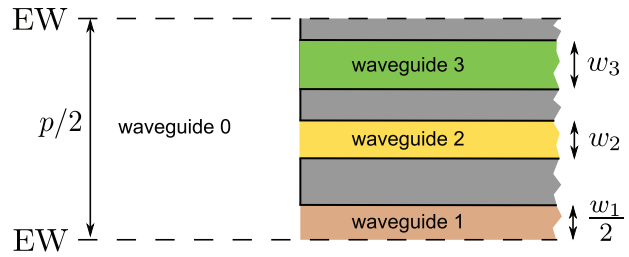

Fig. 16. (a) Generalized waveguide discontinuity problem associated with the half unit cell of a structure with five slits per period and symmetric unit cell.

since the irreducible problem is the complete unit cell. As for the admittances that define the elements of the equivalent circuit, according to (33) it is obtained

$$
\begin{aligned}
\bar{Y}_{i i} & =\frac{4}{p} \sum_{n=1}^{\infty} \xi_{n}^{(0)}\left[\tilde{f}_{n}^{(i)} \cos \left(k_{n} h_{i}\right)\right]^{2} \\
\bar{Y}_{12} & =\frac{4}{p} \sum_{n=1}^{\infty} \xi_{n}^{(0)} \tilde{f}_{n}^{(1)} \tilde{f}_{n}^{(2)} \cos \left(k_{n} h_{1}\right) \cos \left(k_{n} h_{2}\right)
\end{aligned}
$$

for the symmetrical case in Fig. 15(a), and

$$
\begin{aligned}
\bar{Y}_{i i} & =\frac{2}{p} \sum_{n=1}^{\infty} \xi_{n}^{(0)}\left[\tilde{f}_{n}^{(i)}\right]^{2} \\
\bar{Y}_{12} & =\frac{2}{p} \sum_{n=1}^{\infty} \xi_{n}^{(0)} \tilde{f}_{n}^{(1)} \tilde{f}_{n}^{(2)} \cos \left[k_{n}\left(h_{2}-h_{1}\right)\right]
\end{aligned}
$$

for the nonsymmetrical case in Fig. 15(b).

\section{APPENDIX B}

This appendix presents the generalization of the circuit model to structures with more than two slits in the irreducible unit-cell or half unit-cell problem. Consider, for instance, a structure with five slits per period distributed symmetrically within the unit cell. The corresponding generalized waveguide discontinuity associated to the half unit-cell problem is depicted in Fig. 16. By following the same line of reasoning as in Section II-A, (21) for the voltages at the ports naturally becomes

$$
V_{0}=V_{1}+V_{2}+V_{3}
$$




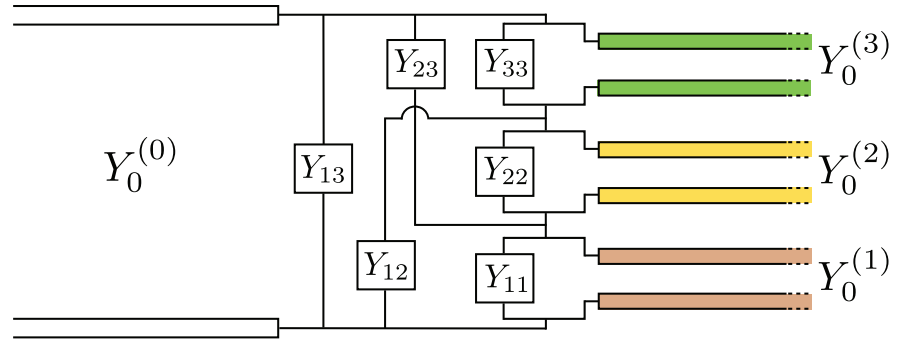

Fig. 17. Topology of the equivalent circuit for a structure in which the irreducible unit cell (or half unit cell) problem has three slits.

and for the current at the input port we now have the following three equations with a similar structure as (25) and (29):

$$
\begin{aligned}
& I_{0}=\left[\bar{Y}_{11}+Y_{0}^{(1)}\right] V_{1}+\bar{Y}_{12} V_{2}+\bar{Y}_{13} V_{3} \\
& I_{0}=\bar{Y}_{12} V_{1}+\left[\bar{Y}_{22}+Y_{0}^{(2)}\right] V_{2}+\bar{Y}_{23} V_{3} \\
& I_{0}=\bar{Y}_{13} V_{1}+\bar{Y}_{23} V_{2}+\left[\bar{Y}_{33}+Y_{0}^{(3)}\right] V_{3} .
\end{aligned}
$$

Now consider the topology shown in Fig. 17, which is the natural extension of the topology previously derived in Section II-A. In this equivalent circuit we can distinguish three coupling orders: a first coupling order represented by the $Y_{12}$ and $Y_{23}$ admittances, which accounts for the interaction between adjacent slits (nearest neighbors); a second-order coupling represented by $Y_{13}$ (second nearest neighbor interaction); and a zeroth-order coupling (self reaction) associated with the $Y_{i i}$ admittances. The current flowing downward through a longitudinal cut placed at the height of the transmission line corresponding to slit 1 can be written as

$$
I_{0}=Y_{13} V_{0}+Y_{12}\left(V_{1}+V_{2}\right)+\left[Y_{11}+Y_{0}^{(1)}\right] V_{1} .
$$

Similarly, the current flowing through longitudinal cuts at slits 2 and 3 are

$$
\begin{aligned}
I_{0}= & Y_{13} V_{0}+Y_{12}\left(V_{1}+V_{2}\right)+Y_{23}\left(V_{2}+V_{3}\right) \\
& +\left[Y_{22}+Y_{0}^{(2)}\right] V_{2} \\
I_{0}= & Y_{13} V_{0}+Y_{23}\left(V_{2}+V_{3}\right)+\left[Y_{33}+Y_{0}^{(3)}\right] V_{3} .
\end{aligned}
$$

Substituting (64) into these last three equations and identifying them with (65)-(67), respectively, it is obtained

$$
\begin{aligned}
& Y_{13}=\bar{Y}_{13} \\
& Y_{12}=\bar{Y}_{12}-Y_{13} \\
& Y_{23}=\bar{Y}_{23}-Y_{13} \\
& Y_{11}=\bar{Y}_{11}-Y_{12}-Y_{13} \\
& Y_{22}=\bar{Y}_{22}-Y_{23}-Y_{12}-Y_{13} \\
& Y_{33}=\bar{Y}_{33}-Y_{23}-Y_{13} .
\end{aligned}
$$

In other words, the admittance $Y_{i j}$ of a given element in the circuit is given by the corresponding barred admittance, $\bar{Y}_{i j}$, minus the admittances of all the elements of higher order coupling that cover the $i j$ element between their connections.
At the light of the above derivation, the generalization to a compound grating whose irreducible unit-cell or half unitcell problem comprises $N_{\mathrm{s}}$ slits is rather straightforward. The topology of the circuit model is the natural extension of that in Fig. 17 with $N_{\mathrm{s}}$ coupling levels (from 0 to $N_{\mathrm{s}}-1$ ). The barred admittances are defined as

$$
\bar{Y}_{i j}=\frac{2}{p} \sum_{n=1}^{\infty} \xi_{n}^{(0)} \tilde{f}_{n}^{(i)} \tilde{f}_{n}^{(j)} \cos \left[k_{n}\left(h_{i}-h_{j}\right)\right]
$$

for structures with a nonsymmetrical unit cell, and

$$
\bar{Y}_{i j}=\frac{4}{p} \sum_{n=1}^{\infty} \xi_{n}^{(0)} \tilde{f}_{n}^{(i)} \tilde{f}_{n}^{(j)} \cos \left(k_{n} h_{i}\right) \cos \left(k_{n} h_{j}\right)
$$

for structures with a symmetrical unit cell (in this case, if the unit cell has an odd number of slits, in the above formula the $h$ parameter of the central slit is taken as zero, and its $w$ parameter corresponds to half its width). The admittances of the elements in the equivalent circuit are given by $(i \leq j)$

$$
Y_{i j}=\bar{Y}_{i j}-\sum_{n, m} Y_{n m}
$$

with

$$
n \leq i ; m \geq j ; \quad(n, m) \neq(i, j) .
$$

\section{REFERENCES}

[1] J. Fraunhofer, "Kurtzer bericht von den resultaten neuerer versuche über die sesetze des lichtes, und die theorie derselbem," Annu. D, Phys., vol. 74, pp. 337-378, 1823.

[2] R. W. Wood, "On a remarkable case of uneven distribution of light in a diffraction grating spectrum," Proc. Phys. Soc. Lond., vol. 18, pp. 269-275, Jun. 1902.

[3] L. Rayleigh, "Note on the remarkable case of diffraction spectra described by prof. Wood," Philos. Mag., vol. 14, no. 79, pp. 60-65, 1907.

[4] L. Rayleigh, "On the dynamical theory of gratings," Proc. R. Soc. Lond., vol. 79, pp. 399-416, Aug. 1907.

[5] T. W. Ebbesen, H. J. Lezec, H. F. Ghaemi, T. Thio, and P. A. Wolff, "Extraordinary optical transmission through sub-wavelength hole arrays," Nature, vol. 391, pp. 667-669, Feb. 1998.

[6] F. J. García-de-Abajo, "Colloquium: Light scattering by particle and hole arrays," Rev. Mod. Phys., vol. 79, pp. 1267-1290, Oct.-Dec. 2007.

[7] C. Genet and T. W. Ebbesen, "Light in tiny holes," Nature, vol. 445, pp. 39-46, Jan. 2007.

[8] F. J. García-Vidal, L. Martín-Moreno, T. W. Ebbesen, and L. Kuipers, "Light passing through subwavelength apertures," Rev. Mod. Phys., vol. 82, pp. 729-787, Jan.-Mar. 2010.

[9] R. Gordon, A. G. Brolo, D. Sinton, and K. L. Kavanagh, "Resonant optical transmission through hole-arrays in metal films: Physics and applications," Laser Photon. Rev., vol. 4, pp. 311-335, Mar. 2010.

[10] J. A. Porto, F. J. García-Vidal, and J. B. Pendry, "Transmission resonances on metallic gratings with very narrow slits," Phys. Rev. Lett., vol. 83, no. 14, pp. 2845-2848, Oct. 2010.

[11] M. M. J. Treacy, "Dynamical diffraction explanation of the anomalous transmission of light through metallic gratings," Phys. Rev. B, Condens. Matter, vol. 66, Nov. 2002, Art. no. 195105.

[12] V. G. Sologub, V. P. Schestopalov, and G. G. Polovnikov, "Diffraction of electromagnetic waves on the grating with narrow slits," J. Tech. Phys., vol. 37, pp. 667-679, Apr. 1967.

[13] V. G. Sologub and V. P. Schestopalov, "The resonance phenomena at the diffraction of the h-polarized waves on the gratings of metal bars," J. Tech. Phys., vol. 38, pp. 667-679, Sep. 1968. 
[14] F. Medina, F. Mesa, and D. C. Skigin, "Extraordinary transmission through arrays of slits: A circuit theory model," IEEE Trans. Microw. Theory Techn., vol. 58, no. 1, pp. 105-115, Jan. 2010.

[15] A. Alù, G. D’Aguanno, N. Mattiucci, and M. J. Bloemer, "Plasmonic brewster angle: Broadband extraordinary transmission through optical gratings," Phys. Rev. Lett., vol. 106, Mar. 2011, Art. no. 123902.

[16] R. Rodríguez-Berral, C. Molero, F. Medina, and F. Mesa, "Analytical wideband model for strip/slit gratings loaded with dielectric slabs," IEEE Trans. Microw. Theory Techn., vol. 60, no. 12, pp. 3908-3918, Dec. 2012

[17] C. Qiu, S. Li, R. Chen, B. Hou, F. Li, and Z. Liu, "Deep subwavelength electromagnetic transparency through dual metallic gratings with ultranarrow slits," Phys. Rev. B, Condens. Matter, vol. 87, May 2013, Art. no. 205129.

[18] Y. Gong, X. Liu, K. Li, J. Huang, J. J. Martínez, D. Rees-Whippey, S. Carver, L. Wang, W. Zhang, T. Duan, and N. Copner, "Coherent emission of light using stacked gratings," Phys. Rev. B, Condens. Matter, vol. 87, May 2013, Art. no. 205121.

[19] T. L. Zinenko, M. Marciniak, and A. I. Nosich, "Accurate analysis of light scattering and absorption by an infinite flat grating of thin silver nanostrips in free space using the method of analytical regularization," IEEE J. Sel. Top. Quantum Electron., vol. 19, no. 3, May/Jun. 2013, Art. no. 9000108

[20] O. V. Shapoval, A. I. Nosich, and J. Ctyrokyb, "Resonance effects in the optical antennas shaped as finite comb-like gratings of noble-metal nanostrips," Proc. SPIE, vol. 8781, pp. 87810U-(1-8), 2013

[21] X. Zhou, J. Fang, D. Yang, X. Zhao, B. Tang, and Z. Liu, "Optical transmission through compound gold surface relief slit arrays," Opt. Exp., vol. 22, pp. 1085-1092, Jan. 2014.

[22] J. Zhou and J. Guo, "Transition from a spectrum filter to a polarizer in a metallic nano-slit array," Sci. Rep. U.K., vol. 4, Jan. 2014, Art. no. 3614.

[23] C. Tardieu, T. Estruch, G. Vincent, J. Jaeck, N. Bardou, S. Collin, and R. Haidar, "Extraordinary optical extinctions through dual metallic gratings," Opt. Lett., vol. 40, pp. 661-664, Feb. 2015.

[24] S.-H. Chang and Y.-L. Su, "Mapping of transmission spectrum between plasmonic and nonplasmonic single slits. I: Resonant transmission," J. Opt. Soc. Amer. B, Opt. Phys., vol. 32, pp. 38-44, Jan. 2015.

[25] S.-H. Chang and Y.-L. Su, "Mapping of transmission spectrum between plasmonic and nonplasmonic single slits. II: Nonresonant transmission,” J. Opt. Soc. Amer. B, Opt. Phys., vol. 32, pp. 45-51, Jan. 2015.

[26] O. V. Shapoval, "Comparison of refractive-index sensitivities of optical-mode resonances on a finite comb-like grating of silver nanostrips," IEEE J. Quantum Electron., vol. 51, no. 4, Apr. 2015, Art. no. 7200108.

[27] J. B. Pendry, L. Martín-Moreno, and F. J. García-Vidal, "Mimicking surface plasmons with structured surfaces," Science, vol. 305, pp. 847-848, Aug. 2004

[28] A. P. Hibbins, B. R. Evans, and J. R. Sambles, "Experimental verification of designer surface plasmons," Science, vol. 308, pp. 670-672, Apr. 2005.

[29] R. E. Collin, Field Theory of Guided Waves. Piscataway, NJ, USA: IEEE Press, 1991.

[30] F. Medina, F. Mesa, and R. Marqués, "Extraordinary transmission through arrays of electrically small holes from a circuit theory perspective," IEEE Trans. Microw. Theory Techn., vol. 56, no. 12, pp. 3108-3120, Dec. 2008

[31] M. García-Vigueras, F. Mesa, F. Medina, R. Rodríguez-Berral, and J. L. Gómez-Tornero, "Simplified circuit model for metallic arrays of patches sandwiched between dielectric slabs under arbitrary incidence," IEEE Trans. Antennas Propag., vol. 60, no. 10, pp. 4637-4649, Oct. 2012.

[32] R. Rodríguez-Berral, F. Medina, F. Mesa, and M. García-Vigueras, "Quasi-analytical modeling of transmission/reflection in strip/slit gratings loaded with dielectric slabs," IEEE Trans. Microw. Theory Techn., vol. 60 , no. 3, pp. 405-418, Mar. 2012.

[33] C. Molero, R. Rodríguez-Berral, F. Mesa, and F. Medina, "Analytical circuit model for 1-D periodic T-shaped corrugated surfaces," IEEE Trans. Antennas Propag., vol. 62, no. 2, pp. 794-803, Feb. 2014.

[34] F. Mesa, M. García-Vigueras, F. Medina, R. Rodríguez-Berral, and J. R. Mosig, "Circuit model analysis of frequency selective surfaces with scatterers of arbitrary geometry," IEEE Antennas Wireless Propag. Lett., vol. 14, pp. 135-138, 2015.
[35] F. Mesa, R. Rodríguez-Berral, M. García-Vigueras, F. Medina, and J. R. Mosig, "Simplified modal expansion to analyze frequency selective surfaces: An equivalent circuit approach," IEEE Trans. Antennas Propag., to be published.

[36] F. Costa, A. Monorchio, and G. Manara, "An overview of equivalent circuit modeling techniques of frequency selective surfaces and metasurfaces," ACES J., vol. 29, no. 14, pp. 960-976, Dec. 2014.

[37] S. A. Masalov and Y. K. Sirenko, "The solution of the problem of the plane wave diffraction on the knife-type grating with compound structure of a period," (in Russian) Radioteknica Elektron., vol. 23, no. 3, pp. 481-487, 1978.

[38] A. N. Fantino, S. I. Grosz, and D. C. Skigin, "Resonant effects in periodic gratings comprising a finite number of grooves in each period," Phys. Rev. E, Stat. Phys. Plasmas Fluids Relat. Interdiscip. Top., vol. 64, Jun. 2001, Art. no. 016605.

[39] S. I. Grosz, D. C. Skigin, and A. N. Fantino, "Resonant effects in compound diffraction gratings: Influence of the geometrical parameters of the surface," Phys. Rev. E, Stat. Phys. Plasmas Fluids Relat. Interdiscip. Top., vol. 65, May 2002, Art. no. 056619.

[40] D. C. Skigin, V. V. Veremey, and R. Mittra, "Superdirectivity radiation from finite gratings of rectangular grooves," IEEE Trans. Antennas Propag., vol. 47, no. 2, pp. 376-383, Feb. 1999.

[41] D. C. Skigin and R. A. Depine, "Transmission resonances of metallic compound gratings with subwavelength slits," Phys. Rev. Lett., vol. 95, Nov. 2005, Art. no. 217402

[42] A. P. Hibbins, I. R. Hooper, M. I. Lockyear, and J. R. Sambles, "Microwave transmission of a compound gratings," Phys. Rev. Lett., vol. 96, Jun. 2006, Art. no. 257402.

[43] Y. G. Ma, X. S. Rao, G. F. Zhang, and C. K. Ong, "Microwave transmission modes in compound metallic gratings," Phys. Rev. B, Condens. Matter, vol. 76, Aug. 2007, Art. no. 085413.

[44] M. Navarro-Cía, D. C. Skigin, M. Beruete, and M. Sorolla, "Experimental demonstration of phase resonances in metallic compound gratings with subwavelength slits in the milimeter wave regime," Appl. Phys. Lett., vol. 94, Mar. 2009, Art. no. 091107.

[45] I. M. Mandel, A. B. Golovin, and D. T. Crouse, "Analytical description of the dispersion relation for phase resonances in compound transmission gratings," Phys. Rev. A, Gen. Phys., vol. 87, May 2013, Art. no. 053833 .

[46] J. R. Andrewartha, J. R. Fox, and I. J. Wilson, "Resonance anomalies in the lamellar gratings," Opt. Acta, vol. 26, no. 1, pp. 69-89, 1979.

[47] A. Wirgin and A. A. Maradudin, "Resonant enhancement of the electric field in the grooves of bare metallic gratings exposed s-polarized light," Phys. Rev. B, Condens. Matter, vol. 31, no. 8, pp. 5573-5576, Apr. 1985.

[48] D. C. Skigin and R. A. Depine, "Resonant enhancement of the field within a single ground-plane cavity: Comparison of different rectangular shapes," Phys. Rev. E, Stat. Phys. Plasmas Fluids Relat. Interdiscip. Top., vol. 59, no. 3, pp. 3661-3668, Mar. 1999.

[49] A. Khavasi and K. Mehrany, "Circuit model for lamellar metallic gratings in the sub-wavelength regime," IEEE J. Quantum Electron., vol. 47 , no. 10 , pp. $1330-1335$, Oct. 2011.

[50] D. W. Woo, S. J. Muhn, and W. S. Park, "Simple analytical model of propagation through thick periodic slot," IEEE Trans. Antennas Propag., vol. 60, no. 1, pp. 5329-5335, Nov. 2012.

[51] E. Yarmoghaddam, G. K. Shirmanesh, A. Khavasi, and K. Mehrany, "Circuit model for a periodic array of slits with multiple propagating diffracted orders," IEEE Trans. Antennas Propag., vol. 62, no. 8, pp. 4041-4048, Aug. 2014.

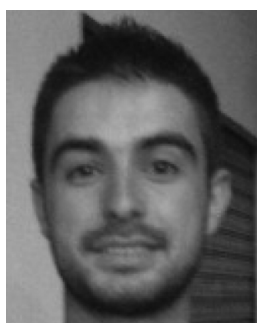

Carlos Molero was born in Las Navas, Seville, Spain, in 1987. He received the Licenciado degree in physics from the University of Sevilla, Seville, Spain, in 2011, and is currently working toward the Ph.D. degree in applied physics at the University of Sevilla.

He is currently with the Department of Applied Physics 1, University of Sevilla.

Mr. Molero was the recipient of a Research Scholarship from the Spanish Ministerio de Economía y Competitividad. 


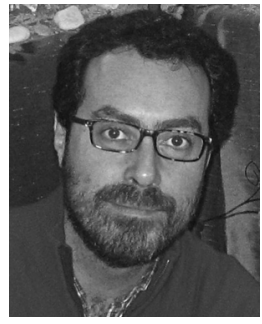

Raúl Rodríguez-Berral was born in Casariche, Seville, Spain, in 1978. He received the M.Sc. (Licenciado) and Ph.D. degrees in physics from the University of Sevilla, Seville, Spain, in 2001 and 2008, respectively.

$\mathrm{He}$ is currently an Associate Professor with the Department of Applied Physics 1, University of Sevilla. His research interests include the study of the spectrum and the excitation of periodic and nonperiodic planar structures and high-frequency circuit modeling.

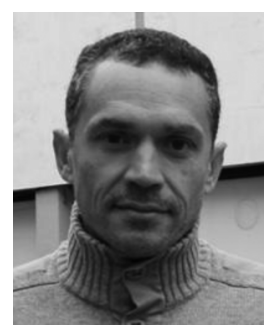

Francisco Mesa (M'93-SM'11-F'14) was born in Cádiz, Spain. He received the Licenciado and Doctor degrees in physics from the University of Sevilla, Seville, Spain.

He is currently a Professor with the Department of Applied Physics, University of Sevilla. His research interests are focused on electromagnetic propagation/ radiation in planar structures

Prof. Mesa has been an Associate Editor for the IEEE TRANSACTIONS ON MicRowAVE THEORY AND TECHNIQUES since 2013.

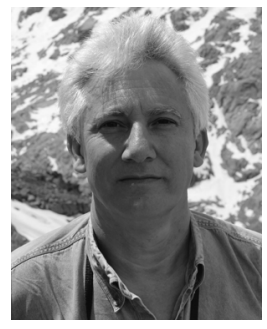

Francisco Medina (M'90-SM'01-F'10) was born in Puerto Real, Cádiz, Spain, in November 1960. He received the Licenciado (M.Sc.) and Doctor (Ph.D.) degrees in physics from the University of Sevilla, Seville, Spain, in 1983 and 1987 respectively.

$\mathrm{He}$ is currently a Professor of electromagnetism with the Department of Electronics and Electromagnetism, University of Sevilla, and Head of the Microwaves Group. His research interests include analytical and numerical methods for planar structures, anisotropic materials, and artificial media modeling.

Prof. Medina has been an Associate Editor for the International Journal of Microwave and Wireless Technologies since 2015. 